Chronic Obstructive Pulmonary Diseases: Journal of the COPD Foundation

\author{
Journal Club
}

\title{
Journal Club - Asthma/Chronic Obstructive Pulmonary Disease Overlap: Fact or Fiction?
}

\author{
Ron Balkissoon, MD, MSc, DIH, FRCPC ${ }^{1}$
}

\begin{abstract}
Abbreviations: Food and Drug Administration, FDA; chronic obstructive pulmonary disease, COPD; asthma/COPD overlap, ACO; longacting muscarinic antagonist, LAMA; immunoglobulin E, IgE; forced expiratory volume in 1 second, FEV1; forced vital capacity, FVC; National Health and Nutrition Examination Survey, NHANES; inhaled corticosteroid, ICS; long-acting beta2-agonist, LABA Citation: Balkissoon R. Journal club - Asthma /chronic obstructive pulmonary disease overlap: fact or fiction? Chronic Obstr Pulm Dis. 2018;5(4):341-350. doi: https://doi.org/10.15326/jcopdf.5.4.2018.0162
\end{abstract}

1 Denver, Colorado

\section{Address correspondence to:}

Ron Balkissoon, MD, MSc, DIH, FRCPC

balkissoonr@njhealth.org

\section{Keywords}

asthma; chronic obstructive pulmonary disease; COPD

\section{Introduction}

With the development of biologics and the advent of "precision medicine" there is great interest in defining phenotypes and indeed endotypes that may identify responders based on clinical characteristics and/ or underlying biological mechanisms. Recently, the Food and Drug Administration (FDA) voted against approval of mepolizumab for use in chronic obstructive pulmonary disease (COPD) stating that there was insufficient evidence to support mepolizumab as an add-on therapy for COPD patients guided by eosinophil levels. The FDA committee pointed to a lack of consensus over the definition and possible relevance of a so-called "eosinophilic COPD phenotype." They pointed to the variability in blood eosinophils making it a challenge to use it as a potential marker to identify appropriate candidates for therapeutic trials. The committee recommended greater research with larger numbers and better patient characterization, including more female patients, (an important recommendation particularly given that women are known to have higher rates of airway hyper-responsiveness than men). Refining the characterization of the asthma/ COPD overlap (ACO) group is key to moving forward in studying the use of biologics in this population. Recent studies have pointed to the benefits of long-acting muscarinic antagonists (LAMA) bronchodilators, originally only indicated for $\mathrm{COPD}$, to be efficacious in some asthma patients (tiotropium has been approved by the FDA for asthma and COPD). ${ }^{1,2}$ Conversely, there have been several trials that have looked at the use of TH-2 pathway biologics typically reserved for asthma patients, (omalizumab, ${ }^{3}$ mepolizumab ${ }^{4-6}$ and benralizumab ${ }^{7}$ ), in COPD patients thought to demonstrate potential overlap features (history of allergies, elevated immunoglobulin E [IgE], high eosinophil counts, asthma history, smoking history, partial reversibility). The various criteria used to define the ACO group have typically utilized some combination of these features. This is the backdrop for reviewing several recent papers that have focused on assessing the prevalence of ACO according to different ACO criteria and/or examined various potential clinical characteristics and/or biomarkers that may lead to greater discrimination in defining the potential endotypes within the ACO group. I present this particular collection of recent studies from this year mostly to demonstrate the highly variable, (and in some instances questionable), criteria for defining ACO patients. With more biologics for airway disease in the pipeline it is critical that we further refine our precision in defining the heterogeneity of the endotypes (or treatable traits) within the ACO group for better patient selection in clinical trials.

Note: Abstracts are presented in their original, published format and have not been edited to match JCOPDF style. 


\section{Abstract 1 \\ Clinical Approach to the Therapy of Asthma-COPD Overlap}

Maselli DJ, Hardin M, Christenson SA, et al. Chest. 2018. pii: S0012-3692(18)31129-2.

doi: https://doi.org/ 10.1016/j.chest.2018.07.028

Over the last few years, there has been a renewed interest in patients with characteristics of both asthma and COPD. Although the precise definition of asthma-COPD overlap (ACO) is still controversial, patients with overlapping features are frequently encountered in clinical practice and may indeed have worse clinical outcomes and increased healthcare utilization than those with asthma or COPD. Therefore, there is a critical need to set a framework for the therapeutic approach of such patients. There are key distinctions in the therapy between asthma and $\mathrm{COPD}$, particularly regarding the initial choice of therapy. However, there is considerable overlap in the use of existing medications for both diseases. Furthermore, novel therapies approved for asthma, such as monoclonal antibodies, may have a role in patients with COPD and ACO. The use of biomarkers, such as peripheral blood eosinophils, exhaled nitric oxide, and serum IgE, may help in selecting appropriate therapies for ACO. In this review, we provide an overview of available treatments for both asthma and COPD and explore their potential role in the treatment of patients with ACO.

\section{Comments}

This is an excellent state of the art review discussing the importance and challenges in defining the ACO group.

\section{Abstract 2 Health Characteristics of Patients With Asthma, COPD and Asthma- COPD Overlap in the NHANES Database}

Llanos JP, Ortega H, Germain G, et al. Int J Chron Obstruct Pulmon Dis. 2018;13:2859-2868.

doi: https://doi.org/10.2147/COPD.S167379

\section{INTRODUCTION:}

Asthma and COPD have overlapping characteristics. As there are limited data on whether asthma-COPD overlap (ACO) represents a distinct condition, this study aimed to determine the similarities and differences of ACO with asthma and COPD.

\section{METHODS:}

US population-based, cross-sectional study using National Health and Nutrition Examination Survey data (2009-2012) compared participants with ACO vs those with asthma or COPD, each as mutually exclusive diseasestates. Demographics, health status, disability/limitations, health care resource utilization, clinical characteristics, and peripheral blood eosinophil counts were analyzed.

\section{RESULTS:}

A total of 1,609, 479, and 299 participants with asthma, COPD, and ACO, respectively, were included. An age-matched asthma subgroup included 299 participants from the asthma group. Compared with asthma and COPD, participants with ACO had worse health status, increased disease burden, and more comorbid conditions. The ACO, vs age-matched asthma subgroup, had lower percent predicted prebronchodilator forced expiratory volume in 1 second ( $82.1 \%$ vs $88.0 \% ; P=0.017$ ). The $\mathrm{ACO}$ group had significantly more asthma attacks in the past year than the age-matched asthma subgroup (49.8\% vs $38.4 \%$; $P<0.001$ ). The ACO group had more participants with postbronchodilator forced expiratory volume in 1 second $<80 \%$ predicted (52.1\%) vs COPD (30.8\%; $P=0.003)$ and more participants with blood eosinophil counts $\geq 400$ cells/ $\mu \mathrm{L}(16.9 \%)$ vs COPD (9.5\%; $P=0.007)$ and the asthma subgroup (6.7\%; $P=0.014)$. 


\section{CONCLUSION:}

The ACO group represents an important subset of patients with chronic respiratory disease with an increased burden of disease over asthma and COPD individually. Early identification of this population will enable appropriate therapeutic interventions in a timely manner.

\section{Comments}

The ACO group in this study were defined as individuals at least 40 years of age and having at least 1 characteristic from the definition for COPD, (spirometric evidence of airflow obstruction forced expiratory volume in 1 second/forced vital capacity [FEV1/FVC] $<0.7$ post bronchodilator or reports of ever being told they had emphysema or chronic bronchitis) or asthma (being at least 12 years of age and reporting having been told by a doctor or health professional that they had or still have asthma or having had an episode of asthma or an asthma attack in the past 12 months). While understanding the challenges of identifying the ACO phenotype based on data available in the National Health and Nutrition Examination Survey (NHANES) data bank, it is important to take this fact into account when interpreting the results. Interestingly the overall results more or less reflect other studies suggesting that ACO patients are more severely impaired than asthma or COPD patients and warrant identification and targeted treatment.

\section{Abstract 3 Concomitant Diagnosis of Asthma and COPD: A Quantitative Study in UK Primary Care}

Nissen F, Morales DR, Mullerova H, et al. Br J Gen Pract. 2018; pii: bjgp 18X699389.

doi: https://doi.org/10.3399/bjgp18X699389

\section{BACKGROUND:}

Asthma and chronic obstructive pulmonary disease (COPD) share many characteristics and symptoms, and the differential diagnosis between the two diseases can be difficult in primary care. This study explored potential overlap between both diseases in a primary care environment.

\section{AIM:}

To quantify how commonly patients with COPD have a concomitant diagnosis of asthma, and how commonly patients with asthma have a concomitant diagnosis of COPD in UK primary care. Additionally, the study aimed to determine the extent of possible misdiagnosis and missed opportunities for diagnosis.

\section{DESIGN AND SETTING:}

Patients with validated asthma and patients with validated COPD in primary care were identified from the UK Clinical Practice Research Datalink (CPRD) in separate validation studies, and the diseases were confirmed by review of GP questionnaires.

\section{METHOD:}

The prevalence of concurrent asthma and COPD in validated cases of either disease was examined based on CPRD coding, GP questionnaires, and requested additional information.

\section{RESULTS:}

In total, 400 patients with COPD and 351 patients with asthma in primary care were identified. Of the patients with validated asthma, 15\% $(n=52)$ had previously received a diagnostic COPD Read code, although COPD was only likely in $14.8 \%$ (95\% confidence interval $[\mathrm{CI}]=11.3$ to 19.0$)$ of patients with validated asthma. More than half (52.5\%, n = 210) of patients with validated COPD had previously received a diagnostic asthma Read code. However, when considering additional evidence to support a diagnosis of asthma, concurrent asthma was only likely in $14.5 \%$ (95\% CI $=11.2$ to 18.3 ) of patients with validated COPD.

\section{CONCLUSION:}

A concurrent asthma and COPD diagnosis appears to affect a relative minority of patients with COPD (14.5\%) or asthma (14.8\%). Asthma diagnosis may be over-recorded in people with COPD.

\section{Comments}

The questionnaires and other diagnostic tools that were used in this study to identify patients with asthma or COPD are quite rigorous and validated. ${ }^{8,9}$ While this may identify the group of patients that carry both diagnoses of asthma and COPD it does not 
necessarily identify the entire ACO population as there are likely patients with underlying asthma biological characteristics without being diagnosed with asthma. The study by Christenson and colleagues that examined the prevalence of a T2 genetic signature in 2 cohorts of COPD individuals in inhaled corticosteroid (ICS)/ long-acting beta2-agonist (LABA) trials found that up to about $20 \%$ had this signature and had the best response to ICS/LABA medications. ${ }^{10}$ Individuals in these trials had to have no prior history of asthma to be included in the studies.

\section{Abstract 4 \\ Overlap of Asthma and Chronic Obstructive Pulmonary Disease in Patients in the United States: Analysis of Prevalence, Features and Subtypes}

Turner RM, Depietro M, Ding B. JMR Public Health Surveill. 2018;4(3):e60.

doi: https://doi.org/10.2196/publichealth.9930

\section{BACKGROUND:}

Although asthma and chronic obstructive pulmonary disease (COPD) are clinically distinct diseases, they represent biologically diverse and overlapping clinical entities and it has been observed that they often cooccur. Some research and theorizing suggest there is a common comorbid condition termed asthma-chronic obstructive pulmonary disease overlap, (ACO). However, the existence of ACO is controversial.

\section{OBJECTIVE:}

The objective of this study is to describe patient characteristics and estimate prevalence, health care utilization, and costs of ACO using claims-based diagnoses confirmed with medical record information.

\section{METHODS:}

Eligible patients were commercial US health plan enrollees; $\geq 40$ years; had asthma, COPD, or ACO; $\geq 3$ prescription fills for asthma/COPD medications; and $\geq 2$ spirometry tests. Records for a random sample of 5000 patients with ACO were reviewed to validate claims-based diagnoses.

\section{RESULTS:}

The estimated ACO prevalence was 6\% (estimated $10,250 / 183,521)$ among 183,521 full study patients. In the claims-based cohorts, the comorbidity burden for ACO was greater versus asthma but similar to COPD cohorts. Medication utilization was higher in ACO versus asthma and COPD. Mean total health care costs were significantly higher for ACO versus asthma but similar to COPD. In confirmed diagnoses cohorts, mean total health care costs (medical plus pharmacy) were lower for ACO versus COPD but similar to asthma (US \$20,035; P=.56). Among confirmed cases, where there was medical record evidence, smoking history was higher in ACO (300/343, 87.5\%) versus asthma cohorts $(100 / 181,55.2 \%)$ but similar to $\operatorname{COPD}(68 / 84,81 \%)$.

\section{CONCLUSIONS:}

ACO had more comorbidities, medication utilization, and costs than patients with asthma or COPD but differences were not seen after confirmation with medical records.

\section{Comments}

In this study, the prevalence rate for ACO is reported as $6 \%$. The similar rate of comorbidities for the ACO population and the COPD population likely reflects that most patients identified as having ACO have carried an initial diagnosis of COPD and that cigarette smoking is associated with a number of the comorbidities (cardiovascular disease, lung cancer, etc.) Note the ACO group had similar smoking rates to the COPD cohort compared to the asthma cohort. It is interesting that the ACO group had health care utilization costs similar to the asthma group and were less than the COPD group. 


\section{Abstract 5 \\ Value of Exhaled Nitric Oxide (FeNO) and Eosinophilia During the Exacerbations of Chronic Obstructive Pulmonary Disease Requiring Hospital Admission}

Río Ramírez MT, Juretschke Moragues MA, Fernández González R, et al. COPD. 2018;1-8.

doi: https://doi.org/10.1080/15412555.2018.1482532

The aim of this study was to analyze whether FeNO levels in acute exacerbation of COPD (AECOPD) with hospital admission have better diagnostic value than eosinophilia in blood, and to evaluate its usefulness in predicting a better clinical response. An observational prospective study of patients with AECOPD was carried out. FeNO determinations were made on arrival at the emergency room (ER), at discharge and during stability 3-6 months after discharge. Co-morbidities, bronchodilators, inhaled (IGC) and systemic (SGC) glucocorticoids, eosinophils, systemic inflammation markers (procalcitonin, C-reactive protein), eosinophil cationic protein, and total IgE were collected. Fifty consecutive patients ( $92 \%$ men, mean age $75 \pm 6$ years) were included in this study. Phenotypes were 26\% Asthma-COPD Overlap Syndrome (ACOS), 42\% chronic bronchitis (CB) and 32\% emphysema. ACOS patients showed significantly higher levels of FeNO (73 ppb) and eosinophils (508 cells/mm3) than the rest (CB: $23 \mathrm{ppb}, 184$ cells/mm3, emphysema: $27 \mathrm{ppb}$, 159 cells/mm3; $\mathrm{p}$ <.05). A significant correlation between FeNO levels measured in ER and eosinophils was observed $(r=0.7 ; p<0.001)$, but not at discharge or in stable phase. No significant association was found with parameters of systemic inflammation and mean stay. In conclusion, the determination of FeNO in AECOPD does not offer advantages over the evaluation of eosinophilia. These parameters rise at arrival in ER, descend at discharge, and remain unchanged in the stable phase. Both present similar diagnostic utility and are able to better identify the ACOS phenotype, which helps select a population that could benefit from a glucocorticoids therapy.

\section{Comments}

This study is interesting in that it suggests that the best time to assess for eosinophilia is right at the time of an acute exacerbation before any treatment is instituted. Waiting until the patient returns to the office after a hospital admission (and probably after an outpatient course of prednisone) will not capture the elevated eosinophil levels that seem to be so elusive to obtain.

\section{Abstract 6 Predictors of Asthma/COPD Overlap in FDNY Firefighters With World Trade Center Dust Exposure: A Longitudinal Study}

\author{
Singh A, Liu C, Putman B, et al. Chest. 2018; pii:
}

S0012-3692(18)31055-31059.

https://doi.org/10.1016/j.chest.2018.07.002

\section{BACKGROUND:}

Previously healthy firefighters with World Trade Center (WTC) dust exposure developed airway disease. Risk factors for irritant-associated asthma/ COPD overlap are poorly defined.

\section{METHODS:}

This study included 2,137 WTC-exposed firefighters who underwent a clinically indicated bronchodilator pulmonary function test (BD-PFT) between 9/11/2001 and 9/10/2017. A post-BD FEV 1 increase of $>12 \%$ and $200 \mathrm{~mL}$ from baseline defined asthma, and a post-BD FEV1/FVC ratio < 0.7 identified COPD cases. Participants who met both criteria had asthma/COPD overlap. Eosinophil levels were measured on screening blood tests performed shortly after $9 / 11 / 2001$ and prior to BDPFT; a subgroup of participants also had serum IgE and 21 cytokines measured $(n=215)$. Marginal Cox regression models for multiple events assessed the associations of eosinophil levels or serum biomarkers with subsequent diagnosis, with age, race, smoking, WTC exposure, first post-9/11 FEV1/FVC ratio, and BMI included as covariates.

\section{RESULTS:}

BD-PFT diagnosed asthma/COPD overlap in 99 
subjects (4.6\%), isolated-asthma in 202 (9.5\%), and isolated-COPD in 215 (10.1\%). Eosinophil concentration $\geq 300$ cells $/ \mu \mathrm{L}$ was associated with increased risk of asthma/COPD overlap (hazard ratio [HR], 1.85; 95\% CI, 1.16-2.95) but not with isolatedasthma or isolated-COPD. Serum IL-4 also predicted asthma/COPD overlap (HR, 1.51 per doubling of cytokine concentration; 95\% CI, 1.17-1.95). Greater IL-21 concentration was associated with both isolated-asthma and isolated-COPD (HRs of 1.73 [95\% CI, 1.27-2.35] and 2.06 [95\% CI, 1.31-3.23], respectively).

\section{CONCLUSIONS:}

In WTC-exposed firefighters, elevated blood eosinophil and IL-4 levels are associated with subsequent asthma/COPD overlap. Disease-specific T-helper cell type 2 biomarkers present years before diagnosis suggest patient-intrinsic predisposition to irritant-associated asthma/COPD overlap.

\section{Comments}

Interesting that the elevated eosinophil count and interleukin-4 counts appeared to predict increased risk of asthma/COPD but not asthma alone. This is an interesting cohort with a unique exposure. Using bronchodilator reversibility to define asthma has its limitations. The biomarker assessment is quite interesting. There remains ongoing controversy as to the differences versus similarities of the underlying pathophysiology of irritant-induced asthma or reactive airways dysfunction syndrome versus traditional occupational asthma associated with latency typically characterized as a T2 sensitization. ${ }^{11-14}$ While the members of this cohort did not have a prior history of airway disease, there remains the question as to whether the exposure caused the asthma or ACO or were there pre-existing T2 features that predisposed them to developing these conditions. In the absence of pre-exposure evaluation of these markers this question cannot be answered from this study.

\section{Abstract 7 Asthma and COPD Overlap in Women: Incidence and Risk Factors}

To T, Zhu J, Gray N, et al. Ann Am Thorac Soc. 2018. doi: https://doi.org/10.1513/AnnalsATS.201802-078OC

\section{RATIONALE:}

Women with asthma are at a high risk of developing COPD, or asthma and COPD overlap syndrome (ACOS) as they age, which is a condition associated with a high mortality rate, low quality of life, and high healthcare costs. However, factors influencing the development of ACOS remain unclear.

\section{OBJECTIVE:}

To quantify the risk of developing COPD in Ontario women with asthma and identify factors that are associated with increased risk.

\section{METHODS:}

Data for Ontario women with asthma who participated in the Canadian National Breast Screening Study from 1980-1985 were linked to health administrative databases and participants were followed from 19922015. A competing risks survival model was used to measure the associations between sociodemographic, lifestyle and environmental risk factors and time to COPD incidence, accounting for death as a competing risk.

\section{RESULTS:}

A total of 4,051 women with asthma were included in the study, of whom 1,701 (42.0\%) developed COPD. The mean age at the study end date was 79 years old. Low education, high body mass index, rurality, and high levels of cigarette smoking were associated with ACOS incidence, while exposure to fine particulate matter (PM2.5), a major air pollutant, was not.

\section{CONCLUSION:}

Individual risk factors appear to play a more significant role in the development of ACOS in women than environmental factors, such as air pollution. Prevention strategies targeting health promotion and education may have the potential to reduce ACOS incidence in this population. 


\section{Comments}

This is a longitudinal follow up of a female cohort over 23 years. Interestingly, the patients who were labeled as having ACO were identified by having had at least 1 COPD hospitalization and/or at least 1 COPD ambulatory care claim. Smoking was not used as part of the criteria to define the ACO group. ACO designation was purely on the basis of claims made that changed the diagnosis from asthma to COPD although, if the COPD diagnosis occurred less than 2 years after their asthma prevalence date, they were excluded. There are the obvious limitations of using classifications based on claims data and how to interpret these results but the analysis according to lifestyle, sociodemographic and exposure variables is very interesting and thoughtprovoking toward intervention and identifying potential high-risk groups.

\section{Abstract 8 \\ Combined Assessment of Serum Periostin and YKL-40 May Identify Asthma-COPD Overlap}

Shirai T, Hirai K, Gon Y, et al. J Allergy Clin Immunol Pract. 2018; pii: S2213-2198(18)30416-1.

doi: https://doi.org/10.1016/j.jaip.2018.06.015

\section{BACKGROUND:}

Asthma-chronic obstructive pulmonary disease (COPD) overlap (ACO) has been proposed as a different diagnosis from asthma and COPD. However, little is known about the role of serum biomarkers in ACO.

\section{OBJECTIVE:}

To evaluate serum periostin, a type 2 biomarker, and serum chitinase-3-like protein 1 (YKL-40), a useful biomarker for COPD, in Japanese patients with asthma, ACO, or COPD, and investigate the role of these biomarkers in identifying ACO.

\section{METHODS:}

Subjects included Japanese patients with asthma ( $n=177), \operatorname{ACO}(n=115)$, or COPD ( $n=61)$. Serum periostin, YKL-40, and total IgE, blood eosinophils, and fractional exhaled nitric oxide were measured and compared among the patients.

\section{RESULTS:}

Serum periostin was high in both asthma and ACO, but not in COPD, whereas serum YKL-40 was high in both COPDand ACO, but not in asthma. Serum periostin levels correlated weakly with eosinophil counts in asthma, ACO, and COPD. Multivariate linear regression analysis revealed that older age, lower body mass index, higher eosinophil counts, higher total $\operatorname{IgE}$, and the absence of the diagnosis of COPD were significantly associated with higher periostin levels. Based on cutoff values derived by receiver operating characteristic analysis (periostin: $55.1 \mathrm{ng} / \mathrm{mL}$; YKL40: $61.3 \mathrm{ng} / \mathrm{mL}$ ), patients were classified into high or low groups. The proportion of patients with both high serum periostin and YKL-40 levels was significantly higher in ACO than in asthma or COPD.

\section{CONCLUSIONS:}

Serum periostin levels were comparable between asthma and ACO, whereas YKL-40 was comparable between ACO and COPD. Combined assessment of serum periostin and YKL-40 may identify ACO.

\section{Comments}

This is an interesting study although it is a relatively small sample size. The definition used in this study for ACO was according to the Global Initiative for Asthma guidelines $^{15}$ : history of asthma, older than 40 , post bronchodilator $\mathrm{FEV}_{1}$, $/ \mathrm{FVC}<0.7$ and at least one of the following criteria including $>10$ pack-year history, diffusing capacity/alveolar volume $<80 \%$ predicted or low attenuation area on high resolution computed tomography and/ or 2 of the following criteria (from modifications to Guidelines) history of asthma, blood eosinophil counts $\geq 250$ cells $/ \mu \mathrm{L}$. FeNO $>35 \mathrm{ppb}$ or serum total IgE $>100$. Patients had been on inhaled corticosteroids, LABAs, LAMAs, leukotriene receptor antagonists, theophylline or omalizumab for at least 6 months. Confounding this data is that $14 \%$ of the ACO group were never smokers. There are also major differences of the proportion of females in the asthma group (71\%) versus in the COPD (20\%) and ACO groups (21\%). Nonetheless, this is a novel and intriguing approach and warrants further study. 


\section{Abstract 9 \\ Differences in Prevalence of Asthma- COPD Overlap According to Different Criteria}

Song JH, Lee CH, Kim DK, et al. Medicine (Baltimore). 2018;97(36): e12049.

doi: https://doi.org/10.1097/MD.0000000000012049

Asthma and chronic obstructive pulmonary disease (COPD) are common chronic airway diseases. Overlap in the clinical features of these 2 diseases is observed in many cases, and thus, the concept of asthmaCOPD overlap (ACO) has recently been proposed. However, the definition of $\mathrm{ACO}$ and the clinical significance remains to be determined. We evaluated the prevalence and risk of acute exacerbation in ACO among Korean COPD patients as defined by modified Spanish criteria and American Thoracic Society (ATS) Roundtable criteria. The prevalence of ACO was $47.7 \%(660 / 1383)$ by modified Spanish criteria and $1.9 \%(26 / 1383)$ by ATS Roundtable criteria. ACO, regardless of criteria, did not significant affect the exacerbation risk during at least 1-year followup period. Substantial discrepancies were found in the prevalence and outcome of ACO according to different diagnostic criteria, which would compromise implementation of $\mathrm{ACO}$ before the definition is established.

\section{Comments}

These are rather sobering results. The 2 sets of criteria ${ }^{16,17}$ have been thoughtfully and rigorously formulated and have been utilized in various studies. It is worth noting that in this study many individuals did not have eosinophil counts, IgE values or age of diagnosis data which would obviously limit the ability to categorize all participants accurately. The number of patients meeting the American Thoracic Society criteria was so low that it makes it difficult to achieve significant statistical power for the comparisons with the number meeting the Spanish criteria.

\section{Abstract 10 \\ Differences in Airway Structural Changes Assessed by Three- Dimensional Computed Tomography in Asthma and Asthma-COPD Overlap}

Niwa M, Fujisawa T, Karayama M, et al . Ann Allergy Asthma Immunol. 2018; pii: S1081-1206(18)30666-5. doi: https://doi.org/10.1016/j.anai.2018.08.006

\section{BACKGROUND:}

Asthma- COPD overlap (ACO) is a clinical phenotype sharing features of asthma and COPD. Multidetector row computed tomography (MDCT) can be used to evaluate the airway structure; however, differences between asthma and ACO seen on MDCT are poorly understood.

\section{OBJECTIVE:}

To investigate the difference in airway structural between asthma and ACO using MDCT in patients with clinical asthma.

\section{METHODS:}

Sixty-four patients with asthma were allocated to an asthma group (neversmokers and ex-smokers with a smoking history of $<10$ pack-years) or an ACO group (patients with a $\geq 10$-pack-year smoking history and FEV1/ FVC <0.7). The asthma group was further divided into patients with airflow limitation (AL; FEV1/FVC <0.7) and those without AL. Wall thickness (WT) and airway inner luminal area in the third-generation to fifth-generation bronchi were evaluated using MDCT in both study groups and in 29 healthy controls.

\section{RESULTS:}

There were 43 patients in the asthma group (20 with AL, 23 without AL) and 16 in the ACO group. Patients with asthma and ACO had significantly greater WT than the healthy controls. WT in the third-generation bronchi was significantly greater in the ACO group than in the asthma group. The ACO group and the asthma with AL group were matched for age, disease duration, FEV1/FVC. WT in the third-generation bronchi was still greater in the ACO group than in the asthma with AL group. 


\section{CONCLUSION:}

Patients with ACO have a thicker airway wall than those with asthma, suggesting that airway remodeling is more prominent in ACO than in asthma.

\section{Comments}

Very small sample size. I am not sure if this approach will be a very practical tool to use to clinically phenotype/endotype patients with asthma versus ACO but it certainly is consistent with other studies that have identified that ACO patients have more fixed airflow obstruction than asthma patients.

\section{Abstract 11 \\ Combined Value of Exhaled Nitric Oxide and Blood Eosinophils in Chronic Airway Disease: The Copenhagen General Population Study}

Çolak Y, Afzal S, Nordestgaard BG, Marott JL, Lange P. Eur Respir J. 2018;52(2). doi: https://doi. org/10.1183/13993003.00616-2018

We investigated whether the combination of increased exhaled nitric oxide fraction (FeNO) level and blood eosinophil count had an additive value in chronic airway disease in the general population. We included 4677 individuals aged 20-100 years from the Copenhagen General Population Study. Based on pre- and post-bronchodilator spirometry, selfreported asthma and smoking history, participants were subdivided into healthy never-smokers ( $n=1649)$, healthy ever-smokers ( $n=1581)$, asthma ( $n=449)$, chronic obstructive pulmonary disease (COPD) $(n=404)$, asthma-COPD overlap (ACO) $(n=138)$ and nonspecific airflow limitation $(\mathrm{n}=456)$. Compared to individuals with $F_{\text {eNo }}<25 \mathrm{ppb}$ and blood eosinophils $<0.3 \times 10^{9}$ cells $\cdot \mathrm{L}^{-1}$, age- and sex-adjusted odds ratios (95\% CI) for wheezing were 1.54 (1.29-1.84) for individuals with $F_{\text {eNo }} \geq 25 \mathrm{ppb}$ or blood eosinophils $\geq 0.3 \times 10^{9}$ cells $\cdot \mathrm{L}^{-1}$ and 2.14 (1.47-3.10) for individuals with $F_{\text {eNO }} \geq 25 \mathrm{ppb}$ and blood eosinophils $\geq 0.3 \times 10^{9}$ cells $\cdot \mathrm{L}^{-1}$ Corresponding odds ratios were 1.13 (0.911.41 ) and 1.83 (1.20-2.79) for sputum production, 1.54 (1.22-1.94) and 3.26 (2.16-4.94) for asthma, $1.03(0.80-1.32)$ and $0.67(0.36-1.27)$ for $C O P D$ and 1.32 (0.88-1.96) and 2.14 (1.05-4.36) for ACO. Among individuals reporting respiratory symptoms, predicting the type of chronic airway disease did not differ between the two biomarkers and did not improve by combining them. Combination of $F_{\mathrm{eNO}}$ and blood eosinophils may have an additive value in characterising chronic airway disease in the general population but still needs to be investigated further with regard to clinical application

\section{Comments}

In this small study the ACO group (n=138) was defined as those individuals who showed fixed airflow limitation $\left(\mathrm{FEV}_{1} / \mathrm{FVC}<0.7\right)$ and self-reported asthma or with pre- and post- bronchodilator $\mathrm{FEV}_{1} / \mathrm{FVC}<0.7$ and who despite no self-reported asthma have significant $\mathrm{FEV}_{1}$ reversibility of $\geq 12 \%$ and $200 \mathrm{ml}$. Unfortunately, the investigators did not document the use of oral steroids and/or inhaled steroids in the different groups and whether this may have influenced the FeNO values and blood eosinophils (blood eosinophils are primarily reduced by oral corticosteroid use). Overall, blood eosinophils appeared to have a slightly greater discriminating power than exhaled nitric oxide with regard to various respiratory symptoms. 


\section{References}

1. Peters SP, Kunselman MA, Icitovic N, et al. Tiotropium bromide step-up therapy for adults with uncontrolled asthma. $N$ Engl $J$ Med. 2010;363(18):1715-1726.

doi: https://doi.org/10.1056/NEJMoa 1008770

2. Shedd GC, Blenis RC. Tiotropium for asthma: a summary of current guidelines and a case study. J Am Assoc Nurse Pract. 2018;30(8):460-463.

doi: https://doi.org/10.1097/JXX.0000000000000053

3. Maltby S, Gibson PG, Powell H, McDonald VM. Omalizumab treatment response in a population with severe allergic asthma and overlapping COPD. Chest. 2017;151(1):78-89. doi: https://doi.org/10.1016/j.chest.2016.09.035

4. Xia Y, Li W, Shen H. Mepolizumab for eosinophilic COPD [letter]. N Engl J Med. 2018;378(7):680-681. doi: https://doi.org/10.1056/NEJMc1715454

5. ShahlaviMonavvar P, Mobasher-Jannat A. Mepolizumab for eosinophilic COPD [letter]. N Engl J Med. 2018;378(7):681. doi: https://doi.org/10.1056/NEJMc1715454

6. Sciurba, FC, Bradford ES, Pavord ID. Mepolizumab for eosinophilic COPD [letter]. N Engl J Med. 2018;378(7):681-683. doi: https://doi.org/10.1056/NEJMc1715454

7. Brightling CE, Bleeker ER, Panettieri RA, et al. Benralizumab for chronic obstructive pulmonary disease and sputum eosinophilia: a randomised, double-blind, placebo-controlled, phase 2a study. Lancet Respir Med. 2014;2(11):891-901. doi: https://doi.org/10.1016/S2213-2600(14)70187-0

8. Quint JK, Müllerova H, DiSantostefano RL, et al. Validation of chronic obstructive pulmonary disease recording in the Clinical Practice Research Datalink (CPRD-GOLD). BMJOpen. 2014;4(7): e005540. doi: https://doi.org/10.1136/bmjopen-2014-005540

9. Nissen F, Quint JK, Wilkinson S, Müllerova H, Smeeth L, Douglas IJ. Validation of asthma recording in electronic health records: protocol for a systematic review. BMJ Open. 2017;7(5): e014694. doi: https://doi.org/10.1136/bmjopen-2016-014694

10. Christenson SA, Steiling K, van den Berge M, et al. AsthmaCOPD overlap. Clinical relevance of genomic signatures of type 2 inflammation in chronic obstructive pulmonary disease. Am J Respir Crit Care Med. 2015;191(7):758-766. doi: https://doi.org/10.1164/rccm.201408-14580C

11. Tarlo SM, Broder I. Irritant-induced occupational asthma. Chest.1989;96(2):297-300.

doi: https://doi.org/10.1378/chest.96.2.297

12. Tarlo SM. Workplace irritant exposures: do they produce true occupational asthma? Ann Allergy Asthma Immunol. 2003;90(5 Suppl 2):19-23.

doi: https://doi.org/10.1016/S1081-1206(10)61643-2
13. Burge PS, Moore VC, Robertson AS. Sensitization and irritantinduced occupational asthma with latency are clinically indistinguishable. Occup Med (Lond). 2012;62(2):129-133. doi: https://doi.org/10.1093/occmed/kqr211

14. Banks DE. Workplace irritant exposures: do they produce true occupational asthma? Curr Opin Allergy Clin Immunol. 2001;1(2):163-168.

doi: https://doi.org/10.1097/00130832-200104000-00008

15. Global Initiative for Asthma (GINA). Global Strategy for Asthma Management and Prevention, 2018. GINA website. https://ginasthma.org/\# Published March 2018. Accessed October 2018.

16. Cosio BG, Soriano JB, López-Campos JL, et al. Defining the Asthma-COPD overlap syndrome in a COPD cohort. Chest. 2016; 149(1):45-52.

doi: https://doi.org/10.1378/chest.15-1055

17. Sin DD. Asthma-COPD overlap syndrome: what we know and what we don't. Tuberc Respir Dis (Seoul). 2017'80(1):11-20. doi: https://doi.org/10.4046/trd.2017.80.1.11 